\title{
Les défis de la gestion du diabète chez les personnes itinérantes : étude qualitative avec la méthode photovoix
}

\author{
Rachel B. Campbell MPP, Matthew Larsen BÉd, Anna DiGiandomenico BA, Marleane A. Davidson, \\ Gillian L. Booth MD MSc, Stephen W. Hwang MD MSP, Kerry A. McBrien MD MSP, David J.T. Campbell MD PhD
}

Citation : CMAJ 2020 July 12;193:E1034-41. doi : 10.1503/cmaj.202537-f

Voir la version anglaise de l'article ici : www.cmaj.ca/lookup/doi/10.1503/cmaj.202537; voir le commentaire connexe en français au www.cmaj.ca/lookup/doi/10.1503/cmaj.210963-f, et en anglais au www.cmaj.ca/lookup/doi/10.1503/cmaj.210963

\section{RÉSUMÉ}

CONTEXTE : La recherche sur les défis de la prise en charge du diabète chez les personnes itinérantes qui en sont atteintes n'a pas tendance à prendre en considération le point de vue des personnes touchées. Nous avons utilisé une approche de recherche participative avec la communauté pour explorer ces défis.

MÉTHODES : Nous avons recruté des cochercheurs ayant une connaissance expérientielle de l'itinérance et du diabète. Les chercheurs principaux leur ont offert une formation en recherche et ont préparé le terrain avec eux pour ce projet. Les cochercheurs ont collectivement choisi d'utiliser la méthode photovoix pour illustrer la difficulté de bien s'alimenter quand on est en situation d'itinérance et explorer en quoi cet écueil affecte plus largement la gestion du diabète. Après une formation en photographie et en éthique, les cochercheurs ont pris des photos en lien avec les objectifs du projet et rédigé des récits connexes au moyen de techniques de rédaction inspirée par des photos. Les chercheurs principaux ont analysé les photos et les récits, et ils en ont dégagé des thèmes qui se sont précisés lors de discussions de groupe.

RÉSULTATS : Les 8 cochercheurs étaient atteints de diabète de type 2 (diagnostiqué de 18 mois à 23 ans auparavant) et avaient vécu en situation d'itinérance pendant des périodes allant de 8 mois à 12 ans. Nous avons dégagé 4 thèmes à partir de 17 photos et récits produits. L'itinérance affecte grandement la santé émotionnelle et mentale des personnes, ce qui nuit à leur capacité de bien gérer leur diabète. Les aliments servis dans les refuges sont rarement nutritifs ou appétissants. L'obtention d'une forme de logement peut faciliter la prise en charge du diabète en créant un environnement stable qui favorise l'autonomie, mais les coûts et le manque de connaissances sont des obstacles à la préparation de repas sains. L'itinérance complique aussi l'accès aux professionnels de la prise en charge du diabète et aux médicaments d'ordonnance.

INTERPRÉTATION : Les images et les récits associés permettent de dresser un tableau frappant, complet et fidèle des défis auxquels sont confrontées les personnes en situation d'itinérance qui essaient de gérer leur diabète. Comprendre ces défis est la première étape qui permettra aux intervenants et aux décideurs de répondre aux besoins de cette population.
$\mathbf{L}$ es patients diabétiques en situation d'itinérance ont souvent de la difficulté à gérer les tâches d'autogestion en raison des multiples défis médicaux et sociaux auxquels ils sont confrontés ${ }^{1}$. Cela inclut le manque d'argent pour acheter des médicaments ou des fournitures pour la glycémie, l'insécurité alimentaire et l'accès difficile aux aliments sains, les barrières sociales et les préjugés qui nuisent à l'accès aux soins ${ }^{2}$, les obstacles à l'atteinte au bien-être émotionnel découlant du stress associé à l'absence d'hébergement stable, ainsi que la difficulté à trouver un endroit où conserver les médicaments et les fournitures pour le traitement du diabète. Dans bien des cas, ces obstacles contribuent à la présence d'une glycémie sous-optimale ${ }^{1}$. Il a d'ailleurs été démontré que les hospitalisations et les complications du diabète sont substantiellement plus élevées chez les personnes en situation d'itinérance ${ }^{3-5}$.

D'autres études ont évalué l'impact de l'itinérance sur le diabète ${ }^{1,6-8}$, mais elles se sont jusqu'ici peu intéressées au point de vue des personnes touchées ${ }^{9}$. Pourtant, la recherche impliquant 
les patients et les citoyens présente une solide feuille de route pour ce qui est de l'adaptation des programmes et de la prestation des services ${ }^{10}$. Les interventions conçues pour les patients atteints du diabète qui font face à des inégalités sociales donnent souvent des résultats renversants, et la participation de la communauté à la conception des interventions est un élément clé des études qui ont donné des résultats positifs ${ }^{11}$. Nous avons voulu utiliser une approche de recherche participative avec la communauté appelée photovoix pour mieux illustrer de quelle façon l'itinérance affecte la prise en charge du diabète.

\section{Méthodes}

L'objectif de la recherche participative avec la communauté est de faire participer des membres de la communauté à la conception de l'étude, à la définition des questions de recherche et à la collecte, à l'analyse et à l'interprétation des données ${ }^{12,13}$. Les membres de la communauté sont très investis ${ }^{14}$ et perçus comme des cochercheurs avec qui les savoirs sont cocréés plutôt que comme de simples participants. Leur expérience concrète est valorisée et leur participation pleine et entière est jugée cruciale ${ }^{15}$. Pour cette étude, les cochercheurs ont formé un comité (Clients with Diabetes Action Committee) dont les membres se sont réunis sur une base bihebdomadaire pour partager leurs connaissances, établir leurs priorités et entreprendre la recherche en fonction de ces priorités.

\section{Participants}

Les membres du groupe ont été recrutés de novembre 2018 à janvier 2019 au moyen d'affiches posées sur des babillards publics et dans des refuges et avec l'aide de groupes communautaires de Toronto. Nous avons recruté des personnes qui vivaient ou avaient vécu en situation d'itinérance alors qu'elles étaient atteintes du diabète. Nous avons adopté une définition large de l'itinérance, soit l'absence d'un hébergement stable, sécuritaire, permanent ou approprié, y compris le fait de vivre provisoirement dans un refuge, dans un refuge d'urgence ou dans la rue. Nous avons inclus des personnes atteintes de diabète de type 1 ou de type 2 autorapporté, et nous avons exclu celles qui ne présentaient que des facteurs de risque de diabète. Nous avons exclu les personnes qui maîtrisaient mal l'anglais et celles qui présentaient d'importants problèmes de santé mentale actifs. D'autres détails sur la formation du groupe sont présentés ailleurs ${ }^{16}$.

Le groupe s'est réuni régulièrement dans un local communautaire pendant 2 heures toutes les 2 semaines. Les membres du groupe ont eu droit à un repas, à des honoraires et à des titres de transport à la fin de chaque réunion. Le groupe s'est montré très motivé, et la participation globale a été de $82 \%$ sur une période de 7 mois.

\section{Modèle d'étude}

Les chercheurs ont présenté aux cochercheurs communautaires un éventail d'approches et de méthodes quantitatives et qualitatives. Les membres du groupe ont exprimé un intérêt pour la méthode photovoix, qui est un outil qualitatif bien caractérisé au moyen duquel les cochercheurs " réalisent et illustrent leurs forces et leurs préoccupations personnelles et sociales, et favorisent le dialogue critique et la compréhension des enjeux individuels et sociaux par le biais de discussions de groupe inspirées par des photos ${ }^{17}$. Depuis sa mise en œuvre initiale par Wang, au milieu des années $1990^{18}$, la méthode photovoix a été utilisée dans divers contextes médicosociaux ${ }^{19}$, en général dans le but de faire entendre la voix des personnes défavorisées ou mal desservies sous l'angle théorique de l'émancipation et de l'autonomisation ${ }^{20}$. La méthode photovoix utilise de manière originale les images comme source d'information pour illustrer et expliciter le point de vue des participants. Cette approche met particulièrement à contribution l'influence potentielle des images sur les politiques à partir de cadres théoriques qui permettent de définir les problèmes de santé, et le rôle des images et des médias dans la production culturelle ${ }^{17,18}$. La méthode photovoix donne aux cochercheurs un plus grand contrôle sur les conclusions de l'étude et, par conséquent, elle s'adapte bien à la recherche participative avec la communauté.

\section{Établissement des objectifs de recherche}

Les questions de recherche ont été préparées par la technique de schématisation conceptuelle, qui est un exercice participatif approfondi d'établissement des priorités échelonné sur plusieurs séances ${ }^{21}$. Le processus est décrit en détail ailleurs ${ }^{22}$. En résumé, les cochercheurs ont participé à des séances de remue-méninges guidées où ils devaient répondre à la question «quels sont les impacts positifs ou négatifs de l'itinérance sur le diabète? » Les cochercheurs ont formulé plusieurs réponses, qu'ils ont ensuite regroupées en catégories. Chaque énoncé a été évalué par les membres du groupe en fonction de son impact sur la gestion du diabète. À la suite de ce processus, le groupe a convenu que le plus grand défi de la gestion du diabète en situation d'itinérance est de bien s'alimenter ${ }^{22}$, ce qui a mené à l'établissement de l'objectif de recherche suivant : prendre une photo qui illustre la difficulté de bien se nourrir quand on est diabétique et en situation d'itinérance.

L'alimentation était la priorité numéro 1 commune à tout le groupe, mais les cochercheurs ont aussi relevé d'autres priorités individuelles. Pour se donner plus de latitude, l'équipe s'est donné un deuxième objectif de recherche élargi, formulé comme suit : prendre une photo qui illustre en quoi l'itinérance facilite ou freine l'autogestion du diabète.

\section{Collecte des données}

Les membres du groupe ont travaillé individuellement et collectivement au projet sur une période de 4 mois, incluant 12 séances de groupe. Chaque cochercheur a reçu un appareil photo numérique automatique (Nikon Coolpix A100). Deux séances de groupe, animées par une photographe professionnelle, ont été consacrées à son maniement. Le groupe a passé une partie de 2 autres séances à discuter de divers enjeux éthiques entourant l'utilisation de photos en recherche, y compris la dynamique des rapports de force, les questions de respect de la vie privée et d'intrusion et la représentation ${ }^{23,24}$. Par ailleurs, plusieurs séances ont été consacrées aux principes de narration à partir d'une photo. 
Certains membres du groupe ont pris une photo pour chaque question de recherche, alors que d'autres en ont pris des dizaines. Les cochercheurs ont soumis leurs photos au groupe pour commentaires et discussion sur le choix de la photo qui illustrait le mieux chaque objectif de recherche. Chaque cochercheur avait le dernier mot sur le choix de la photo ou des photos qu'il souhaitait utiliser. Nous avons demandé à tous les cochercheurs d'envisager de signer des formulaires d'autorisation pour chacun des binômes imagerécit, et de préciser comment ils souhaitaient signer (y compris par initiales, pseudonyme ou anonymement). Nous présentons l'œuvre des cochercheurs avec la signature qu'ils ont choisie.

\section{Analyse de données}

Nous avons analysé les photos sélectionnées en 4 étapes. Premièrement, pour chaque photo, les animateurs ont invité le groupe à répondre à une série de 6 questions exploratoires à l'aide de la méthode $\mathrm{SHOWeD}^{25}$ : Que voyez-vous? Que se passet-il ici? En quoi cela a-t-il un lien avec le diabète dans votre vie? En quoi cela a-t-il un lien avec l'itinérance dans votre vie? Pourquoi cela existe-t-il? Que peut-on y faire?

Après avoir réfléchi à ces questions individuellement, chaque cochercheur a partagé ses réflexions avec le groupe avant de rédiger le récit associé à la photo avec l'aide des chercheurs principaux. Les membres du groupe se sont aussi entraidés en tant que pairs rédacteurs, à la demande du photographe.

Les chercheurs principaux (R.B.C., D.J.T.C.) ont dégagé indépendamment des thèmes qui pouvaient former la trame de toutes les photos et de tous les récits, et qui ont ensuite fait l'objet de discussions avec les cochercheurs. Ces derniers ont ensuite formulé leurs impressions sur la façon dont les photos étaient interreliées et sur les points communs et les différences entre les images. Le groupe a collectivement choisi d'intituler le projet Home Sweet Home(less).

Enfin, le fruit des discussions de cette réunion a été intégré à une analyse subséquente réalisée par les chercheurs principaux (R.B.C., D.J.T.C.), qui ont codé tous les récits par analyse thématique inductive à l'aide d'un logiciel de traitement de texte ${ }^{26}$. L'analyse a été guidée par un cadre visant la compréhension du rôle de la situation socioéconomique dans l'évolution du diabète $^{27}$, avec pour objectif de clarifier et de synthétiser les thèmes émanant du travail des cochercheurs.

\section{Transfert des connaissances}

Une fois le travail finalisé, nous avons présenté les photos sélectionnées et leurs récits associés lors d'une exposition publique dans notre établissement de recherche. Les photos et les récits étaient accompagnés des biographies et des portraits de chaque photographe. Les membres du groupe étaient présents pour parler de leurs photos et répondre aux questions. Après son inauguration à Toronto, l'exposition a été présentée dans différents congrès scientifiques et dans des espaces publics pendant 18 jours à 8 endroits différents au Canada. Nous avons essayé d'avoir des membres du groupe présents à chacune des présentations de l'exposition.

\section{Approbation éthique}

Cette étude a été approuvée par les comités d'éthique de la recherche de l'Université de Calgary et de l'Hôpital St. Michael (Unity Health Toronto).

\section{Résultats}

Nous souhaitions initialement constituer un groupe d'environ 12 cochercheurs, mais nous avons finalement formé un groupe de 8 personnes qui répondaient aux critères d'inclusion et qui ont maintenu leur participation régulière. Les membres du groupe avaient reçu leur diagnostic de diabète de type 2 de 18 mois à 23 ans auparavant et avaient vécu en situation d'itinérance ou d'instabilité liée au logement pendant 18 mois à 12 ans (tableau 1). Le groupe était composé de 3 hommes et de 5 femmes, d'origines ethniques et d'âges divers. Au moment où le groupe a commencé à se réunir, 2 cochercheurs avaient obtenu un logement stable, 3 habitaient dans un logement supervisé et 3 vivaient dans des refuges ou dormaient à l'extérieur. La méthode photovoix a mené à la sélection de 17 photos accompagnées de récits sous 4 thèmes principaux. Les photos sont présentées à l'annexe 1 (accessible en anglais au www.cmaj.ca/lookup/ doi/10.1503/cmaj.202537/tab-related-content). Les 4 thèmes étaient les suivants : effets de l'itinérance sur la santé émotionnelle et mentale et sur l'autogestion du diabète; obstacles à une saine alimentation dans les refuges; avantages et difficultés de la prise en charge du diabète après l'obtention d'un logement; et accès à des professionnels des soins du diabète et aux médicaments d'ordonnance.

\section{Effets de l'itinérance sur la santé émotionnelle et mentale, et sur l'autogestion du diabète}

Les cochercheurs ont décrit l'itinérance comme une situation extrêmement stressante qui oblige les personnes touchées à prioriser leurs besoins les plus fondamentaux (figure 1). L'énergie mentale et le temps nécessaire pour trouver un endroit sûr où passer la nuit, trouver de la nourriture et survivre une autre journée laissent peu de place aux tâches moins urgentes comme la gestion de l'alimentation, la prise de médicaments et l'exercice. À propos de la personne anonyme qui était le sujet de sa photo, un cochercheur a écrit : " J'ignore s'il se savait diabétique ou s'il s'en souciait. Mais l'imaginer prendre ses médicaments et surveiller sa glycémie avec diligence est quelque chose de totalement ridicule » (Matt 3). De plus, l'itinérance peut être oppressante et déprimante pour beaucoup. "Lorsque j'étais assise au bord du trottoir à boire, je ne me souciais pas du tout de mon diabète parce que je n'avais pas de domicile à l'époque. J'étais “dérangée”... »(Georgina 2). Les aliments familiers étaient la seule source de joie dans une autre journée déprimante, même s'ils ne constituaient pas un choix santé pour une personne diabétique : "Un grand lait frappé au chocolat et 2 McDoubles, c'est le point fort de ma journée ... » (Matt 1). Les récits des membres du groupe mentionnaient aussi la peur, l'insécurité et l'injustice (" pourquoi ça m'arrive à moi? ») ressenties en lien avec des difficultés rencontrées jour après jour en essayant de répondre à leurs besoins de base. 
Tableau 1 : Caractéristiques des cochercheurs

\section{Caractéristiques}

$N^{\text {bre }}(\%)$ de participants ${ }^{\star}$

Âge, ans

$<45$

$45-64$

$\geq 65$

Genre

Femmes

Homme

Race

Blanche

Non blanche

Situation de logement au début de l'étude

Sans-abri (dormant en refuge ou à l'extérieur)

Hébergement supervisé ou temporaire

Logement précaire or instable

Durée de l'itinérance/instabilité du logement, moyenne (étendue)

2,81 ans ( 8 mois-12 ans)

Type de diabète

Type 1

Type 2

$8(100)$

Durée du diabète, moyenne (étendue)

7,21 ans (18 mois-23 ans)

Traitement du diabète

Hygiène de vie seulement (sans médicaments)

Médicaments non insuliniques seulement

Injections d'insuline et/ou autres médicaments

Professionnels du diabète

Médecins

Soins primaires seulement

Soins spécialisés

Autres professionnels de la santé

Infirmières

$1(12)$

Diététistes

$2(25)$

Pharmaciens

$1(12)$

Complications diabétiques autorapportées

Coronaropathie ou infarctus du myocarde

Thrombose ou accident vasculaire cérébral

$2(25)$

Ulcère du pied (plaies), gangrène, amputation

$1(12)$

Néphropathie diabétique

$1(12)$

Rétinopathie diabétique

1 (12)

Symptômes neuropathiques

$5(62)$

Comorbidités autorapportées

Hypertension

$7(88)$

Hypercholestérolémie

$5(62)$

Obésité

$6(75)$

Apnée du sommeil

$4(50)$

Dépression

$6(75)$

Problèmes d'anxiété

$4(50)$

Psychose

2 (25)

Alcoolisme

$4(50)$

Toxicomanie

$5(62)$

${ }^{*}$ Sauf indication contraire. 


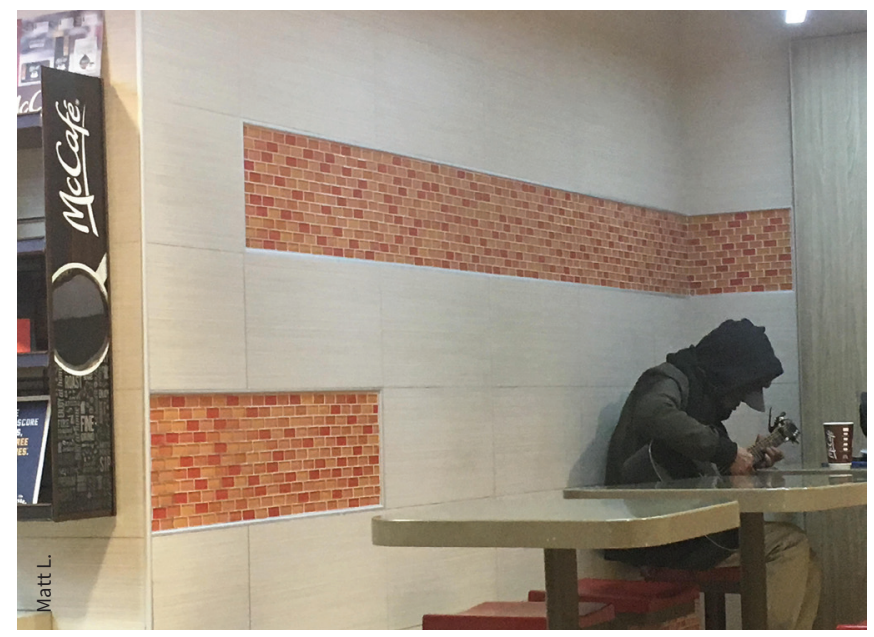

Figure 1 : Always Darkest (L'heure la plus sombre). « Une silhouette solitaire est assise dans un coin du restaurant McDonald's local, jouant de la guitare de manière presque inaudible pour que le personnel ne se fâche pas et ne la chasse pas du restaurant en ce froid matin de la mi-mars. Je me reconnais dans cette photo, sauf qu'au lieu d'être penché sur une guitare, j'ai les yeux rivés sur mon téléphone cellulaire et j'utilise la connexion Wi-Fi du restaurant. Un grand lait frappé au chocolat et 2 McDoubles, c'est le point fort de ma journée, mais ça me tue à petit feu à cause de mon diabète de type 2 . Il y a des McDonald's partout, ouverts 24 heures sur 24, 7 jours sur 7 , à petit prix, et (ce qui compte le plus probablement), c'est un endroit familier. J'y retrouve toujours les mêmes saveurs que dans mes souvenirs. Cette constance est un baume dans le chaos où l'itinérance me plonge jour après jour. Le risque de complications du diabète ne m'effleurait pas l'esprit quand ma vie était un long cauchemar. » (Matt L.).
Obstacles à une saine alimentation dans les refuges Les photos ont montré que manger sainement est extrêmement difficile quand on est dans un refuge (figure 2). Les repas et les collations qu'on y sert ont souvent une piètre valeur nutritive. L'information nutritionnelle des aliments distribués (p. ex., la teneur en glucides) est difficilement accessible. "Ça a l'air bon pour la santé », écrit l'une des membres du groupe, au sujet des aliments qu'on lui a servis. Elle a dû faire " [ses] propres lectures et [ses] propres recherches pour [se] renseigner sur les choix alimentaires santé pour gérer [son] diabète. L'éducation sur le diabète ne fait pas partie de la mission des refuges pour sansabris » (Jasmine 1). Elle a aussi raconté qu'elle devait se procurer elle-même des aliments parce que les repas offerts par le refuge ne convenaient pas.

En outre, selon les membres du groupe, les repas sont souvent peu appétissants. Un cochercheur a écrit qu'il devait se "forcer pour avaler la bouillie » qu'on lui servait au refuge (Matt 2), ce qui vient s'ajouter au fardeau de l'itinérance et donne envie de chercher quelque chose de plus alléchant. Souvent, les seuls autres choix que les repas fournis par les refuges sont des friandises, des boissons gazeuses vendues dans des distributrices « fort populaires » (Ozzy 1), placées ironiquement à proximité d'une publicité pour des ateliers sur la prise en charge du diabète. La plupart des refuges et plusieurs programmes d'hébergement supervisés ne mettent pas de cuisines à la disposition de la clientèle, il est donc impossible de préparer ses propres repas et, par conséquent, quasi impossible d'adopter une alimentation saine.

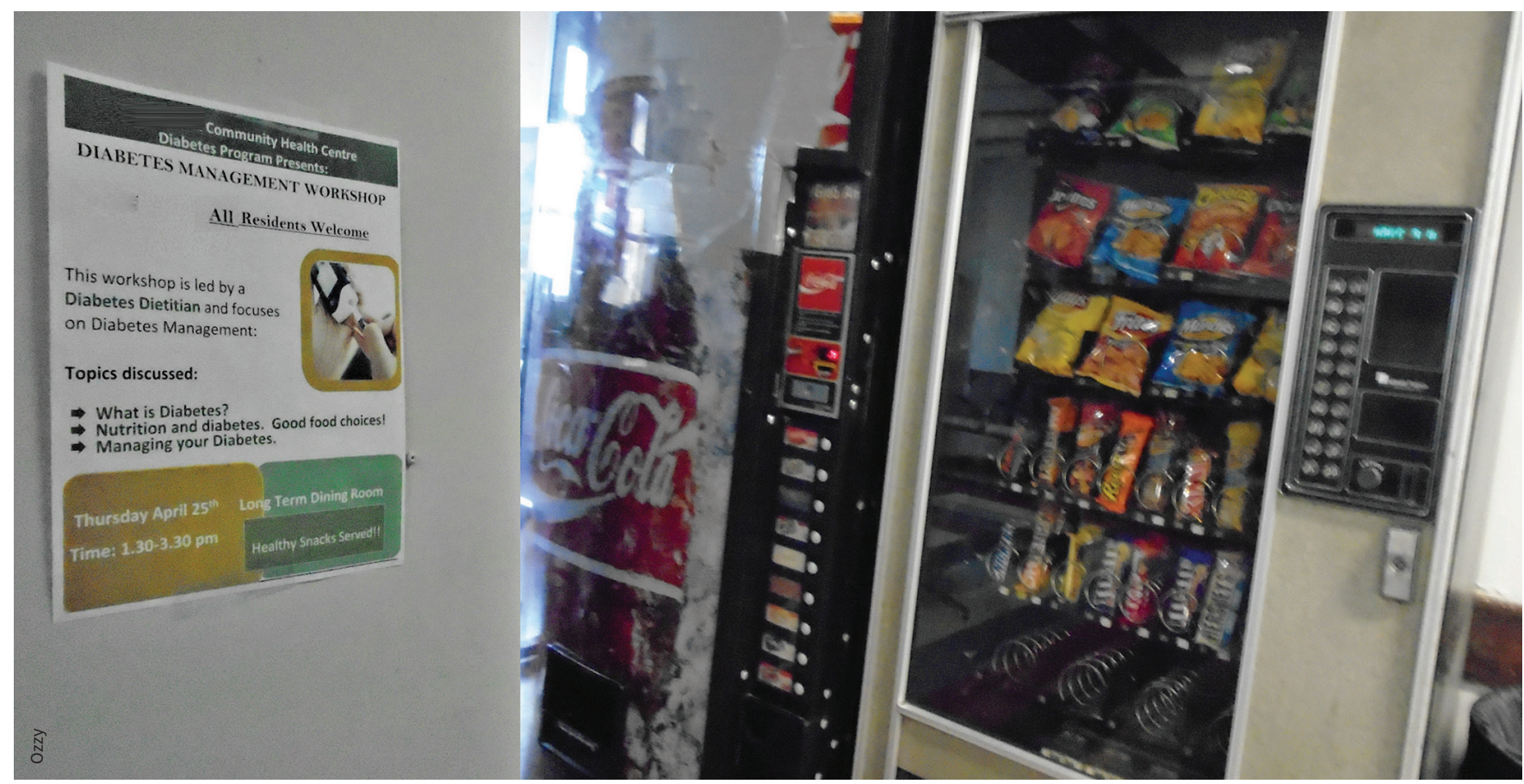

Figure 2 : Contradiction. "Je me suis retrouvé dans un refuge pour sans-abris à Toronto il y a 1 an. Le refuge a récemment affiché le dépliant que vous voyez sur cette photo; cela signifie qu'on y est conscient du problème du diabète. Par contre, à l'arrière-plan, vous voyez 2 distributrices. Celle de gauche propose des boissons gazeuses à forte teneur en sucre, et celle de droite, du chocolat et des friandises. Les 2 distributrices doivent être fort populaires, parce qu'elles doivent être réapprovisionnées chaque dimanche. Comme tout le monde dans le refuge est à risque de développer le diabète, sortez ces machines de là. Si les gens veulent se gaver de friandises, qu'ils sortent pour les trouver. Ou surprenez-nous un de ces jours et remplacez-les par des distributrices d'eau, de pommes ou de sandwichs » (Ozzy). 


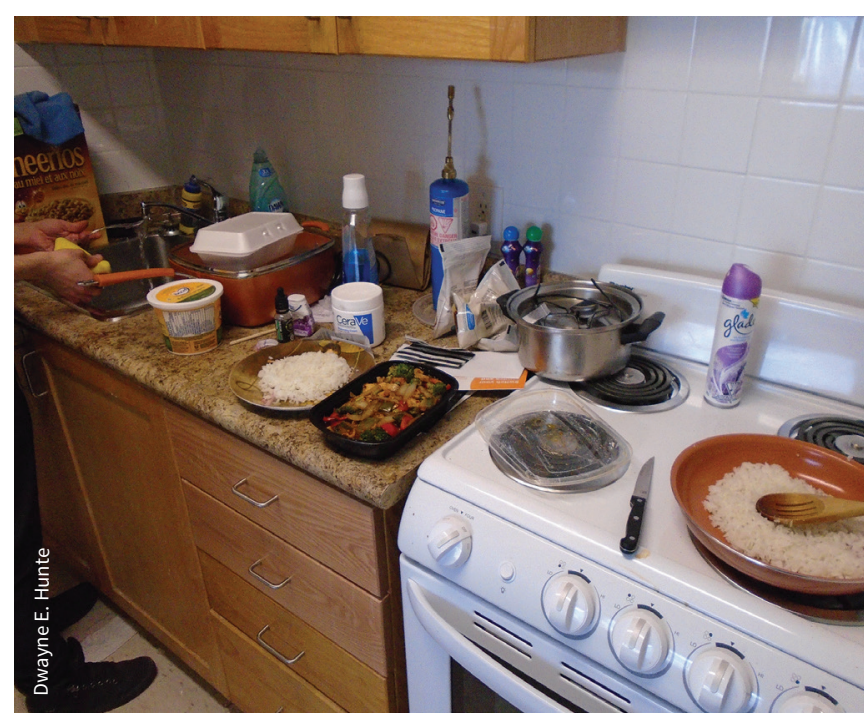

Figure 3 : The Diabetic Wish... (Le souhait d'un diabétique) « Vous voyez ici une cuisine, et pour un diabétique, c'est une bouée de sauvetage. Quand vous êtes dans un refuge, oui... vous avez le choix entre manger un beignet ou une banane, mais soyons honnêtes, qui se préoccupe de ce qu'il mange dans un refuge? Ce qui compte, c'est de savoir si on va me donner à manger ou si je vais aller me coucher le ventre vide. Peu de gens se soucient de savoir cuisiner, mais pour moi, c'est un privilège. $\mathrm{Ne}$ pas avoir à m'inquiéter de la teneur en sucre ou en glucides d'un aliment, pouvoir manger une portion de riz mesurée plutôt que des pâtes, et tous légumes du monde. Être capable de préparer mes repas est une vraie bénédiction : je ne prendrai jamais cela pour acquis, parce que je sais à quel point il est facile de tout perdre, ENCORE UNE FOIS! » (Dwayne E. Hunte).

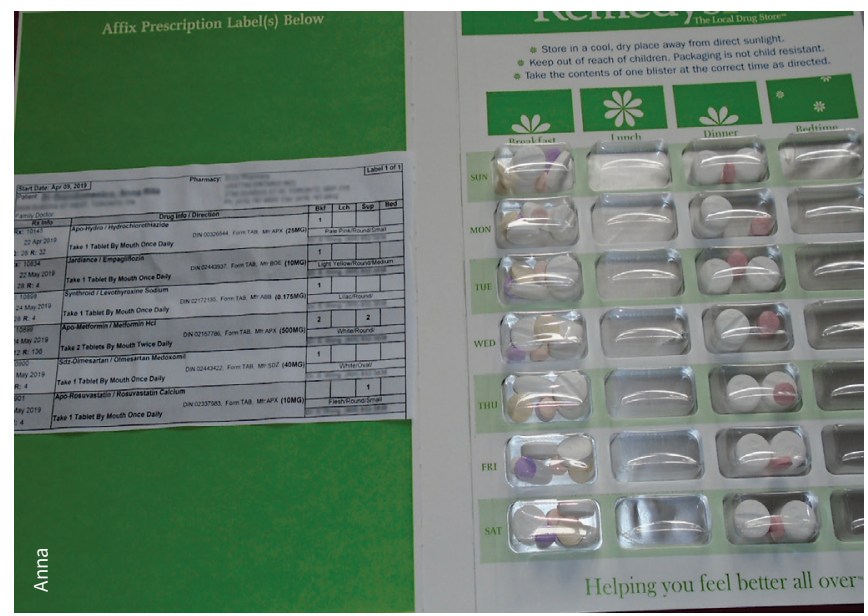

Figure 4 : Patient Pays ... \$97.14 (Et la patiente débourse... 97,14\$) "C'est une blague? C'est une semaine de médicaments. J'imagine que cette semaine, cette fille aura besoin de se contenter de ce qu'il y a dans sa dépense... et ce sera encore des haricots. Alors, expliquez-moi pourquoi? Pourquoi les sans-abris diabétiques doivent choisir cette semaine entre bien manger ou se procurer leurs médicaments pour le diabète? Ensuite, bien sûr, il faut tenir compte de la peur de se faire voler ses médicaments. Alors, maintenant, vous vous passez non seulement de nourriture, mais aussi de vos médicaments. Mais quand quelqu'un agira-t-il contre cette injustice? En tant qu'itinérante diabétique, il faut que je décide si je mange cette semaine ou si je fais renouveler mes ordonnances. Je sais que la bataille s'annonce difficile, mais je ne lâche pas ... je relève le défi, regardez-moi bien aller» (Anna).
Avantages et défis de la prise en charge du diabète après l'obtention d'un logement

L'accès à un logement permanent offre la stabilité et l'autonomie requises pour la prise en charge de cette maladie complexe (figure 3). Une cochercheuse a affirmé que sa vie a changé après qu'elle a obtenu un endroit à elle : "Maintenant que j'ai un toit au-dessus de la tête, je peux conserver mes médicaments chez moi et je n'ai pas à m'inquiéter de les perdre. Je n'ai pas besoin de les garder dans un sac à dos ou dans un sac à ordures que je transporte partout avec moi. Je garde mes médicaments dans leur emballage alvéolé sur la table, et c'est facile pour moi de me rappeler de les prendre. Je n'ai plus peur que quelqu'un me les vole. Mon diabète est assez important, mais ça aurait été bien pire si je n'avais pas obtenu ce logement. » (Georgina 2).

Par contre, l'accès à un logement indépendant s'accompagne d'autres difficultés. Bien manger peut coûter cher pour quelqu'un dont le revenu est limité. Les personnes qui viennent d'obtenir un logement doivent souvent fréquenter les banques alimentaires pour compléter leur épicerie, mais les choix qui y sont offerts sont souvent des aliments à forte teneur glycémique et à faible teneur en protéines et en fibres, comme le montre une photo des aliments provenant d'une banque alimentaire prise par un membre du groupe (annexe 1).

Les personnes qui ne savent pas comment préparer des repas nutritifs peuvent se tourner vers les aliments transformés, qui sont malsains mais facilement accessibles. Plusieurs membres du groupe ont abordé les notions de nutrition, de régime alimentaire et de préparation de repas santé. Une cochercheuse a déclaré avoir participé à des "groupes d'entraide axés sur le diabète pour apprendre comment cuisiner sainement et gérer son diabète par la diétothérapie " (Cat 1). Un autre cochercheur a affirmé " peu de gens se soucient de savoir cuisiner, mais pour moi, c'est un privilège » (Dwayne 2).

\section{Accès aux professionnels des soins du diabète et aux médicaments d'ordonnance}

L'itinérance n'affecte pas que l'aspect alimentaire de la prise en charge du diabète : elle a aussi une incidence sur l'accès aux services de santé et l'observance des traitements prescrits (figure 4). Les cochercheurs ont cité la difficulté pour les itinérants de respecter leurs rendez-vous. Selon eux, rendre ces services plus accessibles en les regroupant ou en amenant les intervenants là où vit la clientèle cible favoriserait le respect des rendez-vous.

Plusieurs cochercheurs ont parlé du coût des médicaments d'ordonnance pour des gens comme eux qui ne sont pas bénéficiaires d'un régime public d'assurance médicaments ou admissibles à de tels régimes. Ceux qui avaient accès à l'assurance médicaments ont pour leur part mentionné certaines restrictions, comme le non-remplacement des médicaments en cas de vol. Au sujet du coût des médicaments d'ordonnance, une cochercheuse a écrit : " en tant qu'itinérante diabétique, il faut que je décide si je mange cette semaine ou si je fais renouveler mes ordonnances » (Anna 2). Parmi les autres défis associés à la 
prise des médicaments, mentionnons le besoin de lieux adéquats où les conserver (en l'absence d'un endroit privé et sécuritaire) et les problèmes d'observance thérapeutique dus à des horaires irréguliers et à l'absence de routine.

\section{Interprétation}

Nous avons sollicité la participation de cochercheurs dans la communauté pour illustrer les nombreux défis associés à la prise en charge du diabète chez les personnes en situation d'itinérance. Notre processus a commencé par une question très ouverte pour explorer le diabète et l'itinérance selon une approche de recherche participative avec la communauté. Même si l'expérience des individus était variée et nuancée, nous avons constaté une certaine uniformité dans les obstacles rencontrés. Nos cochercheurs ont dit que les enjeux liés à l'alimentation et aux aliments étaient leur principale difficulté, mais notre projet photovoix a illustré qu'au-delà du contrôle glycémique, l'alimentation affectait aussi le bien-être mental et émotionnel, et que ces problématiques persistaient souvent malgré l'obtention d'un logement stable. Beaucoup d'autres difficultés liées au diabète n'ont pas été approfondies par nos cochercheurs, car dans leur esprit, elles étaient moins importantes.

Un aspect particulier de la recherche participative avec la communauté est que les projets sont généralement entrepris avec l'objectif explicite de contribuer à modifier significativement les politiques ou les pratiques. Les conclusions de ce projet ont des implications pratiques claires : les personnes en situation d'itinérance qui sont atteintes du diabète auraient besoin de plus de soutien pour mieux s'alimenter et gérer leur diabète, qu'ils soient sans abri ou nouvellement logés. Nos conclusions pourraient avoir des conséquences particulièrement pour les instances subventionnaires, mais aussi pour les refuges, les fournisseurs de repas et les organismes responsables de prodiguer des soins de santé. Nos initiatives de transfert des connaissances ont entre autres pris la forme de présentations dans des organismes locaux et d'expositions de photos dans des congrès universitaires nationaux et internationaux, ainsi que d'activités de défense des intérêts par les cochercheurs. Plusieurs membres du groupe ont pris l'initiative de demander au personnel de leurs refuges et d'autres endroits où ils sont nourris d'offrir des options alimentaires adaptées aux personnes diabétiques. Au moins 2 programmes ou refuges se sont engagés à modifier les repas fournis. Une membre du groupe entretient actuellement un dialogue au sujet de ces enjeux avec son député fédéral.

Même si les défis associés au fait d'être à la fois diabétique et en situation d'itinérance ${ }^{1,2}$ et les solutions potentielles (p. ex., hébergement ${ }^{28,29}$ et amélioration de l'accès aux soins de santé ${ }^{30}$ ) ont déjà été abordés, les photos et les récits créés par des cochercheurs ayant une connaissance expérientielle enrichissent la discussion et lui donnent plus de poids. Très peu d'études sur le diabète ont adopté une approche participative intégrant la voix et le point de vue des personnes touchées ${ }^{9}$. D'autres projets de photovoix ont été réalisés auprès de personnes en situation d'itinérance ${ }^{31}$, incluant 2 qui ont fait état de la difficulté d'accéder à la nourriture ${ }^{32,33}$, mais notre étude s'intéressait plus précisément aux défis liés à la prise en charge du diabète dans le contexte de l'itinérance.

Nous avons recruté pour ce projet un groupe diversifié de cochercheurs de la communauté, qui représentaient un vaste éventail de points de vue et d'expériences. Étant donné que le thème et les questions pour cette recherche ont été établis en partenariat avec les membres du groupe, ces derniers se sont particulièrement investis dans le projet. De plus, étant donné que le projet photovoix était intégré à une initiative de recherche participative basée sur la communauté ${ }^{16}$, le groupe a consacré substantiellement plus de temps à travailler collectivement comparativement à plusieurs autres projets photovoix. La relation entre les chercheurs et les membres du groupe était déjà bien établie avant le début du projet photovoix, et ce lien de confiance a facilité la réalisation de la portion récit. Le fait de consacrer de nombreuses heures aux réunions de groupe a contribué à la rigueur de notre projet. La force de notre étude réside dans sa capacité de présenter un récit convaincant et puissant qui, nous l'espérons, continuera d'inspirer les chercheurs, les cliniciens et les décideurs, afin qu'ils défendent les intérêts de cette population pour lui faciliter l'accès à des aliments sains et à d'autres biens et services essentiels.

\section{Limites}

Une des limites de cette étude est le petit nombre de cochercheurs, qui découle de la taille de l'échantillon de l'étude mère pour ce projet de recherche participative avec la communauté. Il s'agit donc d'un groupe autosélectionné de membres de la communauté qui ont exprimé un intérêt pour le projet. C'est toutefois la petite taille du groupe qui a favorisé son ouverture, ainsi que la richesse et la franchise des discussions, car nous avons pu tisser des liens de confiance entre les cochercheurs et les chercheurs principaux. Le petit nombre de participants a aussi permis aux chercheurs principaux de consacrer du temps à chaque cochercheur, de susciter des récits et leur donner forme. Le processus de recherche participative avec la communauté demandait aux membres du groupe de s'engager à assister à des réunions périodiques sur une période de 6-8 mois. Cette exigence a malheureusement empêché la participation de personnes en situation de crise (p. ex., hébergement instable, violence, problèmes de santé mentale ou physique et dépendances). Une autre limite a été l'absence d'adolescents, de jeunes adultes et de personnes atteintes de diabète de type 1 ; le groupe était néanmoins diversifié du point de vue de l'âge, du sexe, de l'ethnicité, de l'expérience de l'itinérance et du traitement du diabète. Comme c'est le cas pour beaucoup d'autres études qualitatives, le nombre de participants et la nature de la démarche imposent une certaine prudence quand vient le temps d'appliquer les conclusions à d'autres populations et à d'autres environnements, compte tenu des particularités des contextes. Étant donné que le groupe a choisi les priorités et les questions de recherche, ces dernières pourraient ne pas 
concorder avec celles d'autres populations dans d'autres environnements. Finalement, les chercheurs universitaires ont joué un rôle central dans l'analyse des données générées par les cochercheurs, et nous reconnaissons que notre point de vue est influencé par notre expérience et notre situation privilégiée. Nous avons beaucoup travaillé auprès des personnes en situation d'itinérance, mais nous n'avons pas personnellement d'expérience vécue de l'itinérance. Nous avons, dans la mesure du possible, tenté de mettre en valeur la voix des cochercheurs plutôt que d'imposer notre propre perspective.

\section{Conclusion}

Comme l'incidence de l'itinérance ${ }^{34}$ et du diabète de type 2 continue d'augmenter chez les personnes vivant en situation de vulnérabilité sociale ${ }^{35-37}$, nous nous attendons à ce que les enjeux mis en relief par les cochercheurs au cours de cette étude persistent. Cette étude se veut un point de départ pour les décideurs afin qu'ils réfléchissent aux solutions envisageables pour les personnes diabétiques vivant en situation d'itinérance. Finalement, nous espérons que ce projet montrera à d'autres chercheurs du domaine de la santé que la recherche communautaire, faisant par exemple appel à la méthode photovoix utilisée ici, est une entreprise faisable, utile et même essentielle pour comprendre les besoins et les points de vue de cette population.

\section{Références}

1. Hwang SW, Bugeja AL. Barriers to appropriate diabetes management among homeless people in Toronto. CMAJ 2000;163:161-5.

2. Campbell DJ, O'Neill BG, Gibson K, et al. Primary healthcare needs and barriers to care among Calgary's homeless populations. BMC Fam Pract 2015;16:139.

3. Benoit SR, Zhang Y, Geiss LS, et al. Trends in diabetic ketoacidosis hospitalizations and in-hospital mortality - United States, 2000-2014. MMWR Morb Mortal Wkly Rep 2018;67:362-5.

4. Booth GL, Hux JE. Relationship between avoidable hospitalizations for diabetes mellitus and income level. Arch Intern Med 2003;163:101-6.

5. Schlienger J. Some recent figures about diabetes in France [article en français]. Met Dis Med 2009;3:98-9.

6. Axon RN, Gebregziabher M, Dismuke CE, et al. Differential impact of homelessness on glycemic control in veterans with type 2 diabetes mellitus. J Gen Intern Med 2016;31:1331-7.

7. Davachi S, Ferrari I. Homelessness and diabetes: reducing disparities in diabetes care through innovations and partnerships. Can J Diabetes 2012;36: 75-82.

8. Elder NC, Tubb MR. Diabetes in homeless persons: barriers and enablers to health as perceived by patients, medical, and social service providers. Soc Work Public Health 2014;29:220-31.

9. Harris J, Haltbakk J, Dunning T, et al. How patient and community involvement in diabetes research influences health outcomes: a realist review. Health Expect 2019;22:907-20.

10. Crawford MJ, Rutter D, Manley C, et al. Systematic review of involving patients in the planning and development of health care. BMJ 2002;325:1263.

11. Glazier RH, Bajcar J, Kennie NR, et al. A systematic review of interventions to improve diabetes care in socially disadvantaged populations. Diabetes Care 2006;29:1675-88.

12. Israel BA, Schulz AJ, Parker EA, et al. Critical issues in developing and following community-based participatory research principles In: Minkler M, Wallterstein N, editors. Community-based participatory research for health. San Francisco: Jossey-Bass; 2003:56-73.
13. Israel BA, Schulz AJ, Parker EA, et al. Community-based participatory research: policy recommendations for promoting a partnership approach in health research. Educ Health (Abingdon) 2001;14:182-97.

14. Viswanathan M, Ammerman A, Eng E. Community-based participatory research: assessing the evidence. Rockville: Agency for Healthcare Research and Quality; 2004.

15. Israel BA, Schulz AJ, Parker EA, et al. Review of community-based research: assessing partnership approaches to improve public health. Annu Rev Public Health 1998;19:173-202.

16. Campbell D, Campbell R, DiGiandomenico A, et al. The use of a participatory patient engagement research project to meaningfully engage those with lived experience of diabetes and homelessness [préimpression]. medRxiv 2021 Mar. 8. doi: https://doi.org/10.1101/2021.02.26.21252531.

17. Wang C. Photovoice: a participatory action research strategy applied to women's health. J Womens Health 1999;8:185-92.

18. Wang C, Burris MA. Photovoice: concept, methodology, and use for participatory needs assessment. Health Educ Behav 1997;24:369-87.

19. Catalani C, Minkler M. Photovoice: a review of the literature in health and public health. Health Educ Behav 2010;37:424-51.

20. Wang C, Burris MA. Empowerment through photo novella: portraits of participation. Health Educ Q 1994;21:171-86.

21. Trochim WMK. An introduction to concept mapping for planning and evaluation. Eval Program Plan 1989;12:1-16.

22. Grewal E, Campbell R, Booth G, et al. Using concept mapping to prioritize barriers to diabetes care and self-management for those who experience homelessness [préimpression]. Research Square 2021 Mar. 1. doi: https://www.researchsquare. com/article/rs-267860/v1

23. Creighton G, Oliffe JL, Ferlatte 0 , et al. Photovoice ethics: critical reflections from men's mental health research. Qual Health Res 2018;28:446-55.

24. Wang CC, Redwood-Jones YA. Photovoice ethics: perspectives from Flint Photovoice. Health Educ Behav 2001;28:560-72.

25. Wang C, Burris MA. Photovoice: concept, methodology, and use for participatory needs assessment. Health Educ Behav 1997;24:369-87.

26. Braun V, Clarke V. Thematic analysis. In: Cooper H, editor. APA handbook of research methods in psychology. Vol 2. Washington (D.C.): American Psychological Association; 2012.

27. Brown AF, Ettner SL, Piette J, et al. Socioeconomic position and health among persons with diabetes mellitus: a conceptual framework and review of the literature. Epidemiol Rev 2004;26:63-77.

28. Keene D, Henry M, Gormley C, et al. 'Then I found housing and everything changed': transitions to rent-assisted housing and diabetes self-management. Cityscape 2018;20:107-18.

29. Keene DE, Guo M, Murillo S. "That wasn't really a place to worry about diabetes": Housing access and diabetes self-management among low-income adults. Soc Sci Med 2018;197:71-7.

30. Campbell D, Campbell R, Booth G, et al. Innovations in providing diabetes care for individuals experiencing homelessness: an environmental scan. Can J Diabetes 2020; 44:643-50.

31. Seitz C, Strack R. Conducting public health photovoice projects with those who are homeless: a review of the literature. J Soc Distress Homeless 2016;25:33-40.

32. Bredesen J, Stevens M. Using photovoice methodology to give voice to the health care needs of homeless families. Am Int J Contemp Res 2013;3:1-12.

33. Valera P, Gallin J, Schuk D, et al. "Trying to eat healthy": a photovoice study about women's access to healthy food in New York City. Affilia 2009;24:300-14.

34. Everyone counts: highlights. Preliminary results from the second nationally coordinated point-in-time count of homelessness in Canadian communities. Ottawa: Employment and Social Development Canada; 2018.

35. An economic tsunami: the cost of diabetes in Canada. Toronto: Canadian Diabetes Association; 2009.

36. Diabetes in Canada: highlights from the Canadian Chronic Disease Surveillance System. Ottawa: Public Health Agency of Canada; 2017.

37. Lin J, Thompson TJ, Cheng YJ, et al. Projection of the future diabetes burden in the United States through 2060. Popul Health Metr 2018;16:9. 
Intérêts concurrents : Aucun déclaré.

Cet article a été soumis à l'examen des pairs.

Affiliations : Département de médecine (R.B. Campbell, D.J.T. Campbell), Faculté de médecine Cumming de l'Université de Calgary, Calgary, Alb.; Clients with Diabetes Action Committee, MAP Centre for Urban Health Solutions (Larsen, DiGiandomenico, Davidson), Institut du savoir Li Ka Shing, Hôpital St. Michael, Unity Health Toronto; MAP Centre for Urban Health Solutions (Booth, Hwang), Institut du savoir Li Ka Shing, Hôpital St. Michael, Unity Health Toronto; Département de médecine, Faculté de médecine (Booth, Hwang), Université de Toronto, Toronto, Ont.; Départements des sciences de la santé communautaire (McBrien, D.J.T. Campbell), de médecine familiale (McBrien) et de cardiologie (D.J.T. Campbell), Faculté de médecine Cumming de l'Université de Calgary, Calgary, Alb.

Collaborateurs : David Campbell, Rachel Campbell, Matthew Larsen, Anna DiGiandomenico, Marleane Davidson et les autres cochercheurs communautaires ont contribué à la conception du travail. Matthew Larsen, Anna DiGiandomenico et Marleane Davidson ont procédé à la collecte des données sous la supervision de David Campbell et Rachel Campbell, avec les commentaires de Kerry McBrien, Gillian Booth et Stephen Hwang. Matthew Larsen, Anna DiGiandomenico, Marleane Davidson et d'autres cochercheurs communautaires ont analysé les données avec David Campbell et Rachel Campbell. Tous les auteurs et les cochercheurs communautaires ont contribué à l'interprétation des données. Rachel Campbell et David Campbell ont rédigé la première version du manuscrit. Matthew Larsen, Anna DiGiandomenico, Marleane Davidson, Kerry McBrien, Gillian Booth et Stephen Hwang ont contribué substantiellement à la révision critique du manuscrit. Tous les auteurs ont aussi donné leur approbation finale pour la version destinée à être publiée et assument l'entière responsabilité de tous les aspects du travail.
Propriété intellectuelle du contenu : Il s'agit d'un article en libre accès distribué conformément aux modalités de la licence Creative Commons Attribution (CC BY-NC-ND 4.0), qui permet l'utilisation, la diffusion et la reproduction dans tout médium à la condition que la publication originale soit adéquatement citée, que l'utilisation se fasse à des fins non commerciales (c.-à-d., recherche ou éducation) et qu'aucune modification ni adaptation n'y soit apportée. Voir : https://creativecommons. org/licenses/by-nc-nd/4.0/deed.fr.

Financement : Alberta Innovates a contribué au financement de cette étude sous la forme d'une bourse pour cliniciens chercheurs attribuée au $D^{r}$ Campbell, tout comme le Cal Wenzel Family Cardiometabolic Research Fund (département de médecine de la Faculté de médecine Cumming) et le programme de recherche et de formation sur la santé des populations vulnérables (Institut O’Brien de santé publique, Faculté de médecine Cumming), par l'intermédiaire de subventions versées au $D^{r}$ D. Campbell. Les organismes subventionnaires n'ont joué aucun rôle dans la conception ou la conduite de l'étude ni dans la rédaction ou l'approbation du manuscrit en vue de sa publication.

Partage des données : Toutes les données générées dans le cadre de cette étude (images et récits) sont présentées à l'annexe.

Remerciements : Les auteurs aimeraient remercier Linda Monteith pour la formation sur le maniement des appareils photo. Les auteurs remercient aussi Pat O'Campo, Priya Vasa et Janet Parsons pour leurs conseils durant le projet de recherche, et tiennent à souligner la participation du personnel du TD Centre for Learning, qui a accueilli le groupe communautaire. Enfin, ce projet n'aurait pas vu le jour sans le dévouement, l'implication et les talents des cochercheurs de la communauté. Les auteurs les remercient de s'être investis pour aider la population à mieux comprendre leurs luttes.

Accepté : 22 avril 2021

Correspondance : David Campbell, dcampbel@ucalgary.ca 